\title{
Failure properties of fiber bundle models
}

\author{
SRUTARSHI PRADHAN AND BIKAS K. CHAKRABARTI \\ Saha Institute of Nuclear Physics \\ 1/AF Bidhan Nagar, Kolkata 700064, India. \\ E-mail: spradhan@cmp.saha.ernet.in \\ E-mail: bikas@cmp.saha.ernet.in
}

\begin{abstract}
We study the failure properties of fiber bundles when continuous rupture goes on due to the application of external load on the bundles. We take the two extreme models: equal load sharing model (democratic fiber bundles) and local load sharing model. The strength of the fibers are assumed to be distributed randomly within a finite interval. The democratic fiber bundles show a solvable phase transition at a critical stress (load per fiber). The dynamic critical behavior is obtained analytically near the critical point and the critical exponents are found to be universal. This model also shows elastic-plastic like nonlinear deformation behavior when the fiber strength distribution has a lower cutoff. We solve analytically the fatigue-failure in a democratic bundle, and the behavior qualitatively agrees with the experimental observations. The strength of the local load sharing bundles is obtained numerically and compared with the existing results. Finally we map the failure phenomena of fiber bundles in terms of magnetic model (Ising model) which may resolve the ambiguity of studying the failure properties of fiber bundles in higher dimensions.
\end{abstract}

Keywords: fiber bundle; critical behavior; universality; fatigue

\section{Introduction}

Fracture processes in heterogeneous media, initiated by external loading has rich physical and mathematical aspects ${ }^{1}$. Since past decades, people are trying to study this fracture phenomena through different models as well as through direct experiments. The knowledge of the strength of a disordered solid and prior knowledge of its failure properties are of extreme importance in architectural engineering, textile engineering and in construction of any mechanical structure. This knowledge is also required to design material microstructures which can be used to construct highly reliable components. In the context of geophysics the dynamical aspects of fracture process is useful to explain the earthquake phenomena. Some theoretical models like lattice models in various dimensions have been proposed to analyse the details of the fracture phenomena; among these the fiber bundle model is the earliest and the simplest one.

A loaded bundle of fibers represents the various aspects of fracture process through its self-organised dynamics. The fiber bundle model study was initiated by Peirce $^{2}$ in the context of testing the strength of cotton yarns. Since then this 
model has been studied from various points of view. Fiber bundles are of two classes with respect to the time dependence of fiber strength: The 'static' bundles contain fibers whose strengths are independent of time, whereas the 'dynamic' bundles are assumed to have time dependent elements to capture the creep rupture and fatigue behaviors. According to the load sharing rule, fiber bundles are being classified into two groups: Equal load-sharing (ELS) bundles or democratic bundles and local loadsharing (LLS) bundles. In democratic bundles intact fibers bear the applied load equally and in local load-sharing bundles the terminal load of the failed fiber is given equally to all the intact neighbors. The classic work of Daniels ${ }^{3}$ on the strength of the static fiber bundles under equal load sharing (ELS) assumption initiated the probabilistic analysis of the model ${ }^{4-15}$. The distribution of burst avalanches during fracture process is a marked feature of the fracture dynamics and can be observed in ultrasonic emissions during the fracture process. It helps characterizing different physical systems along with the possibility to predict the large avalanches. From a nontrivial probabilistic analysis, Hemmer and Hansen $^{5}$ got power law distribution of avalanches for static ELS bundles, whereas the power law exponent observed numerically for static LLS bundles differs significantly. This observation induces the possibility of presenting loaded fiber bundles as earthquake models ${ }^{6}$. The recent mean field estimate of the 'avalanches' in the ELS bundles ${ }^{8,11}$, gives a new power $\operatorname{law}^{11}$. The phase transition ${ }^{7,8}$ and dynamic critical behavior of the fracture process in such bundles has been established through recursive formulation ${ }^{9,10,11,12}$ of the failure dynamics. The exact solutions ${ }^{10,11,12}$ of the recursion relations suggest universal values of the exponents involved. Attempt has also been made ${ }^{13}$ to study the ELS ans LLS bundles from a single framework introducing a 'range of interaction' parameter which determines the load transfer rule.

Coleman ${ }^{14}$ started working on time dependent bundles under ELS to obtain their life time with steady load. Later, subsequent generalization was made by Phoenix ${ }^{15}$ where both ELS and LLS were considered. Some recent developments ${ }^{16-22}$ show fatigue behavior of ELS bundles considering fluctuation in applied load. Also, introducing noise-induced failure probability of fibers, fatigue behavior is achieved ${ }^{17}$ in a homogeneous fiber bundle under ELS with steady load.

In this report, we give a brief summary of the exactly solvable ${ }^{10,11,12}$ static fiber bundle models under ELS assumptions. This gives the mean field behavior of the failure dynamics, its critical behavior and its universality. An analytic study of fatigue-failure in homogeneous bundle under $\operatorname{ELS}^{17}$ is also discussed. Some modifications and discussions have been added here to correlate the sequential developments. We also show numerically that critical strength of such linear bundles under LLS, vanishes in the large chain limit. This is basically a confirmation of earlier observations ${ }^{22,23}$. A brief magnetic mapping model of fiber bundles is also discussed later. 


\section{Equal load sharing (ELS) bundles}

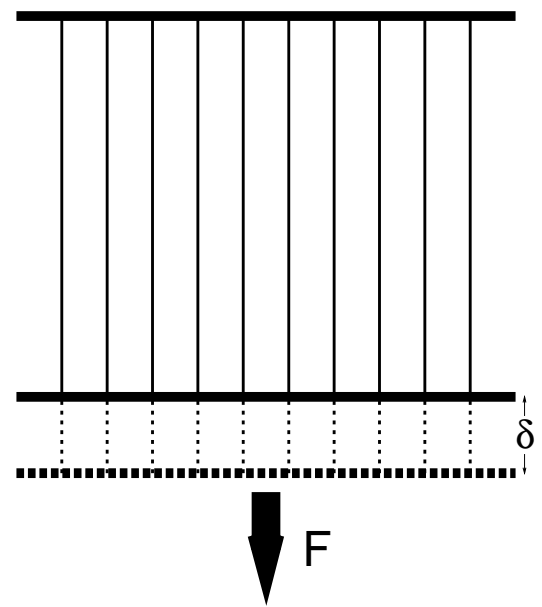

Fig. 1: The fiber bundle consists initially of $N$ fibers attached in parallel to a fixed plate at the top and a movable plate from which a load $F$ is suspended at the bottom. In the ELS model the load $F$ is equally shared by all the intact fibers.

The fiber bundle consists of $N$ fibers, each having identical spring constant $\kappa$. The bundle supports a load $F=N \sigma$ and the breaking threshold $\left(\sigma_{t h}\right)_{i}$ of the fibers are assumed to be different for different fiber $(i)$. ELS model assumes equal load sharing, i.e., the intact fibers share the applied load $F=N \sigma$ equally where $\sigma$ is the initial applied stress (load per fiber). The strength of each of the fiber $\left(\sigma_{t h}\right)_{i}$ in the bundle is given by the stress value it can bear, and beyond which it fails. The strength of the fibers are taken from a randomly distributed normalised density $\rho\left(\sigma_{t h}\right)$ within the interval 0 and 1 such that

$$
\int_{0}^{1} \rho\left(\sigma_{t h}\right) d \sigma_{t h}=1
$$

The equal load sharing assumption neglects 'local' fluctuations in stress (and its redistribution) and renders the model as a mean-field one.

\subsection{Breaking dynamics of the ELS models}

The breaking dynamics starts when an initial stress $\sigma$ (load per fiber) is applied on the bundle. The fibers having strength less than $\sigma$ fail instantly. Due to this rupture, total number of intact fibers decreases and rest of the (intact) fibers have to bear the applied load on the bundle. Hence effective stress on the fibers increases and 
this compels some more fibers to break. These two sequential operations, the stress redistribution and further breaking of fibers continue till an equilibrium is reached, where either the surviving fibers are strong enough to bear the applied load on the bundle or all fibers fail.

This self organised breaking dynamics can be represented by recursion relations in discrete time steps. Let $U_{t}$ be the fraction of fibers in the initial bundle that survive after time step $t$, where time step indicates the number of occurrence of stress redistribution. Then the redistributed load per fiber after $t$ time step becomes

$$
\sigma_{t}=\frac{\sigma}{U_{t}}
$$

and after $t+1$ time steps the surviving fraction of fiber is

$$
U_{t+1}=1-P\left(\sigma_{t}\right)
$$

where $P\left(\sigma_{t}\right)$ is the cumulative distribution of the corresponding density $\rho\left(\sigma_{t h}\right)$ :

$$
P\left(\sigma_{t}\right)=\int_{0}^{\sigma_{t}} \rho\left(\sigma_{t h}\right) d \sigma_{t h}
$$

Now using Eq. (2) and Eq. (3) we can write the recursion relations which show how $\sigma_{t}$ and $U_{t}$ evolve in discrete time:

$$
\sigma_{t+1}=\frac{\sigma}{1-P\left(\sigma_{t}\right)} ; \sigma_{0}=\sigma
$$

and

$$
U_{t+1}=1-P\left(\sigma / U_{t}\right) ; U_{0}=1
$$

The recursion relations (5) and (6) represent the basic dynamics of failure in equal load sharing models. At the equilibrium or steady state $U_{t+1}=U_{t} \equiv U^{*}$ and $\sigma_{t+1}=\sigma_{t} \equiv \sigma^{*}$. This is a fixed point of the recursive dynamics. Eq. (5) and Eq. (6) can be solved at the fixed point for some particular form of $\rho\left(\sigma_{t h}\right)$ and these solutions near $U^{*}$ (or $\sigma^{*}$ ) give the detail features of the failure dynamics of the bundle. 


\subsection{Phase transition and critical behavior for uniform distribution of fiber strength}

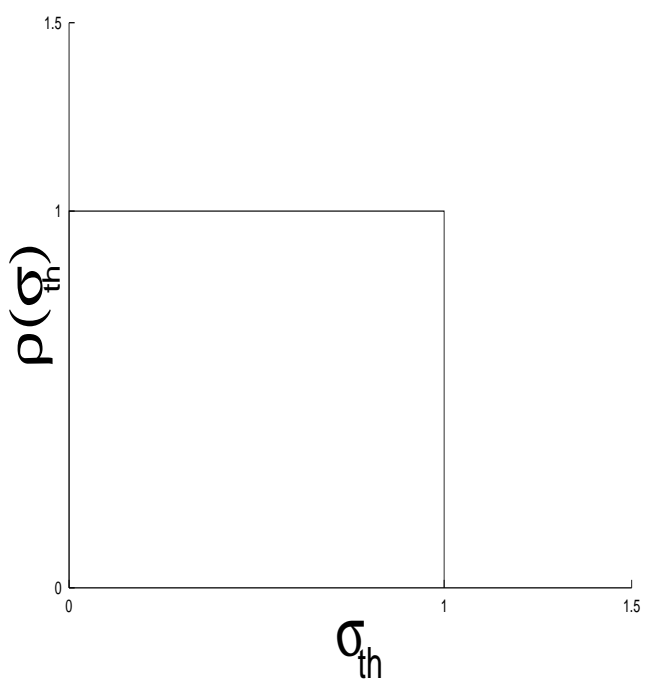

Fig. 2: The simplest model considered here assumes uniform density $\rho\left(\sigma_{t h}\right)$ of the fiber strength distribution up to a cutoff strength.

We choose the uniform density of fiber strength distribution to solve the recursive failure dynamics of democratic bundle. Here, the cumulative distribution becomes

$$
P\left(\sigma_{t}\right)=\int_{0}^{\sigma_{t}} \rho\left(\sigma_{t h}\right) d \sigma_{t h}=\int_{0}^{\sigma_{t}} d \sigma_{t h}=\sigma_{t} .
$$

Therefore $U_{t}$ follows a simple recursion relation (following Eq. (6))

$$
U_{t+1}=1-\frac{\sigma}{U_{t}} .
$$

At the equilibrium state $\left(U_{t+1}=U_{t}=U^{*}\right)$, the above relation takes a quadratic form of $U^{*}$ :

$$
U^{*^{2}}-U^{*}+\sigma=0
$$

The solution is

$$
U^{*}(\sigma)=\frac{1}{2} \pm\left(\sigma_{c}-\sigma\right)^{1 / 2} ; \sigma_{c}=\frac{1}{4} .
$$

Here $\sigma_{c}$ is the critical value of initial applied stress beyond which the bundle fails completely. The solution with $(+)$ sign is the stable one, whereas the one with $(-)$ sign gives unstable solution ${ }^{11,12}$. The quantity $U^{*}(\sigma)$ must be real valued as it has a physical meaning: it is the fraction of the original bundle that remains intact under a fixed applied stress $\sigma$ when the applied stress lies in the range $0 \leq \sigma \leq \sigma_{c}$. Clearly, $U^{*}\left(\sigma_{c}\right)=1 / 2$ (putting $\sigma=\sigma_{c}$ in Eq. 10). Therefore the stable solution can be written as

$$
U^{*}(\sigma)=U^{*}\left(\sigma_{c}\right)+\left(\sigma_{c}-\sigma\right)^{1 / 2} ; \sigma_{c}=\frac{1}{4} .
$$


For $\sigma>\sigma_{c}$ we can not get a real-valued fixed point as the dynamics never stops until $U_{t}=0$ when the bundle breaks completely. It may be noted that the quantity $U^{*}(\sigma)-U^{*}\left(\sigma_{c}\right)$ behaves like an order parameter that determines a transition from a state of partial failure $\left(\sigma \leq \sigma_{c}\right)$ to a state of total failure $\left(\sigma>\sigma_{c}\right)^{11,12}$ :

$$
O \equiv U^{*}(\sigma)-U^{*}\left(\sigma_{c}\right)=\left(\sigma_{c}-\sigma\right)^{\beta} ; \beta=\frac{1}{2} .
$$

(a) At $\sigma<\sigma_{c}$

To study the dynamics away from criticality ( $\sigma \rightarrow \sigma_{c}$ from below), we replace the recursion relation (8) by a differential equation

$$
-\frac{d U}{d t}=\frac{U^{2}-U+\sigma}{U} .
$$

Close to the fixed point we write $U_{t}(\sigma)=U^{*}(\sigma)+\epsilon$ (where $\epsilon \rightarrow 0$ ). This, following Eq. (10), gives $^{10,11}$

$$
\epsilon=U_{t}(\sigma)-U^{*}(\sigma) \approx \exp (-t / \tau)
$$

where $\tau=\frac{1}{2}\left[\frac{1}{2}\left(\sigma_{c}-\sigma\right)^{-1 / 2}+1\right]$. Near the critical point we can write

$$
\tau \propto\left(\sigma_{c}-\sigma\right)^{-\alpha} ; \alpha=\frac{1}{2}
$$

Therefore the relaxation time diverges following a power-law as $\sigma \rightarrow \sigma_{c}$ from below $^{10,11}$.

One can also consider the breakdown susceptibility $\chi$, defined as the change of $U^{*}(\sigma)$ due to an infinitesimal increment of the applied stress $\sigma^{10,11,12}$

$$
\chi=\left|\frac{d U^{*}(\sigma)}{d \sigma}\right|=\frac{1}{2}\left(\sigma_{c}-\sigma\right)^{-\gamma} ; \gamma=\frac{1}{2}
$$

from equation (10). Hence the susceptibility diverges as the applied stress $\sigma$ approaches the critical value $\sigma_{c}=\frac{1}{4}$. Such a divergence in $\chi$ had already been observed in the numerical studies ${ }^{7,9}$.

(b) At $\sigma=\sigma_{c}$

At the critical point $\left(\sigma=\sigma_{c}\right)$, we observe a dynamic critical behavior in the relaxation of the failure process to the fixed point. From the recursion relation (8) it can be shown that decay of the fraction $U_{t}\left(\sigma_{c}\right)$ of unbroken fibers that remain intact at time $t$ follows a simple power-law ${ }^{11,12}$ :

$$
U_{t}=\frac{1}{2}\left(1+\frac{1}{t+1}\right)
$$

starting from $U_{0}=1$. For large $t(t \rightarrow \infty)$, this reduces to $U_{t}-1 / 2 \propto t^{-\delta} ; \delta=1$; a strict power law which is a robust characterization of the critical state. 


\subsection{Universality class of the model}

To check the universality class of the model we have taken two other types of fiber strength distributions: linearly increasing density distribution and linearly decreasing density distribution within the limit 0 and 1 . We solve the recursion equations $(5)$ and (6) in these two cases. We show that while $\sigma_{c}$ changes with different strength distributions, the critical behavior remains unchanged: $\alpha=1 / 2=\beta=\gamma, \delta=1$.

\section{(a) Linearly increasing density of fiber strength}

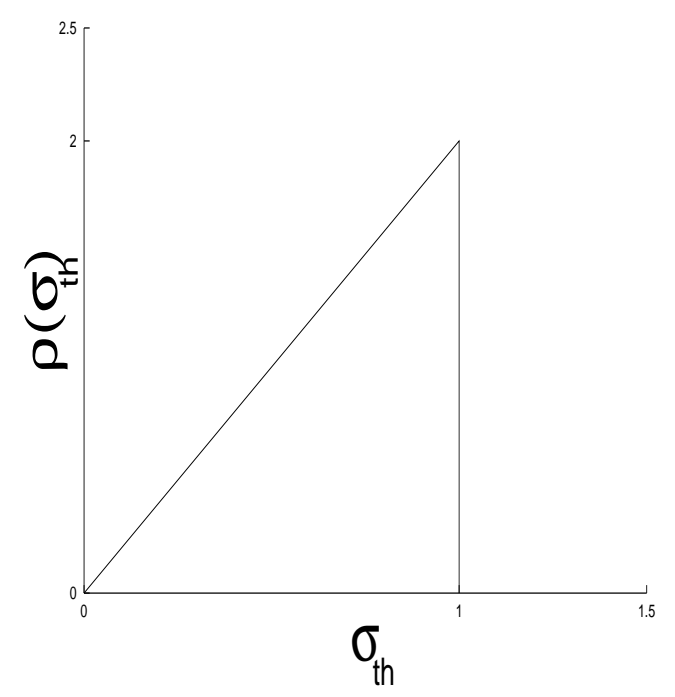

Fig. 3: The linearly increasing density $\rho\left(\sigma_{t h}\right)$ of the fiber strength distribution up to a cutoff strength.

Here, the cumulative distribution becomes

$$
P\left(\sigma_{t}\right)=\int_{0}^{\sigma_{t}} \rho\left(\sigma_{t h}\right) d \sigma_{t h}=2 \int_{0}^{\sigma_{t}} \sigma_{t h} d \sigma_{t h}=\sigma_{t}^{2} .
$$

Therefore $U_{t}$ follows a recursion relation (following Eq. (6))

$$
U_{t+1}=1-\left(\frac{\sigma}{U_{t}}\right)^{2}
$$

At the fixed point $\left(U_{t+1}=U_{t}=U^{*}\right)$, the above recursion relation can be represented by a cubic equation of $U^{*}$

$$
\left(U^{*}\right)^{3}-\left(U^{*}\right)^{2}+\sigma^{2}=0 .
$$

Solving the above equation we get the value of critical stress $\sigma_{c}=\sqrt{4 / 27^{12}}$ which is the strength of the bundle for the above fiber strength distribution. Here, the order parameter can be defined as $U^{*}(\sigma)-U^{*}\left(\sigma_{c}\right)$ and this goes as

$$
O \propto\left(\sigma_{c}-\sigma\right)^{\beta} ; \beta=\frac{1}{2} .
$$


S. Pradhan and B. K. Chakrabarti

The susceptibility diverges as the critical point is approached from below:

$$
\chi=\left|\frac{d U^{*}(\sigma)}{d \sigma}\right| \propto\left(\sigma_{c}-\sigma\right)^{-\gamma} ; \gamma=\frac{1}{2} .
$$

We can also show that for any $\sigma<\sigma_{c}$

$$
U_{t}(\sigma)-U^{*}(\sigma) \approx \exp (-t / \tau)
$$

with

$$
\tau \propto\left(\sigma_{c}-\sigma\right)^{-\alpha} ; \alpha=\frac{1}{2}
$$

and at $\sigma=\sigma_{c}$

$$
U_{t}-U^{*}\left(\sigma_{c}\right) \propto t^{-\delta} ; \delta=1 .
$$

\section{(b) Linearly decreasing density of fiber strength}

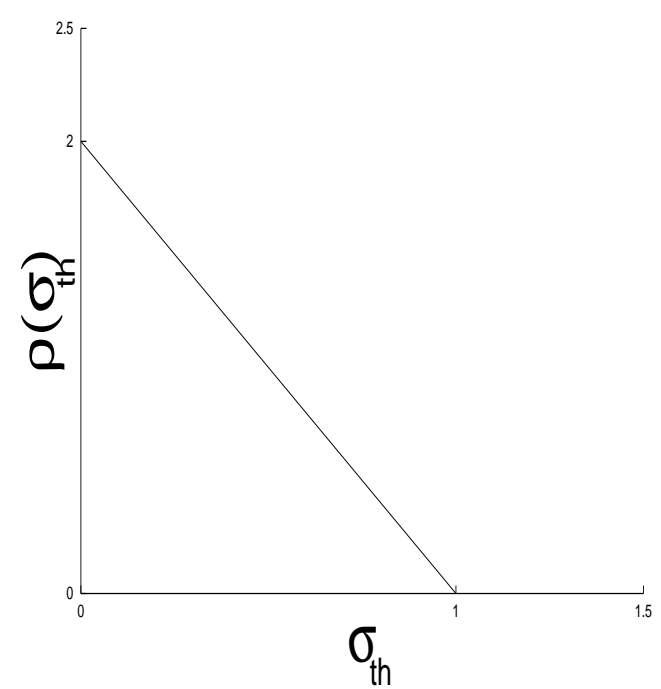

Fig. 4: The linearly decreasing density $\rho\left(\sigma_{t h}\right)$ of the fiber strength distribution up to a cutoff strength.

In this case, the cumulative distribution becomes

$$
P\left(\sigma_{t}\right)=\int_{0}^{\sigma_{t}} \rho\left(\sigma_{t h}\right) d \sigma_{t h}=2 \int_{0}^{\sigma_{t}}\left(1-\sigma_{t h}\right) d \sigma_{t h}=2 \sigma_{t}-\sigma_{t}^{2}
$$

and $U_{t}$ follows a recursion relation (following Eq. (6))

$$
U_{t+1}=1-2 \frac{\sigma}{U_{t}}+\left(\frac{\sigma}{U_{t}}\right)^{2}
$$

At the fixed point $\left(U_{t+1}=U_{t}=U^{*}\right)$, the above recursion relation can be represented by a cubic equation of $U^{*}$

$$
\left(U^{*}\right)^{3}-\left(U^{*}\right)^{2}+2 \sigma U^{*}-\sigma^{2}=0 .
$$


Solution of the above equation suggests the value of critical stress $\sigma_{c}=4 / 27^{12}$ which is the strength of the bundle for the above fiber strength distribution. Also, the order parameter goes as

$$
O \equiv\left[U^{*}(\sigma)-U^{*}\left(\sigma_{c}\right)\right] \propto\left(\sigma_{c}-\sigma\right)^{\beta} ; \beta=\frac{1}{2}
$$

and the susceptibility diverges with the similar power law as in Eqs. (16 and 22) when the critical point is approached from below:

$$
\chi=\left|\frac{d U^{*}(\sigma)}{d \sigma}\right| \propto\left(\sigma_{c}-\sigma\right)^{-\gamma} ; \gamma=\frac{1}{2} .
$$

Here also for any $\sigma<\sigma_{c}$

$$
U_{t}(\sigma)-U^{*}(\sigma) \approx \exp (-t / \tau)
$$

where

$$
\tau \propto\left(\sigma_{c}-\sigma\right)^{-\alpha} ; \alpha=\frac{1}{2}
$$

and at $\sigma=\sigma_{c}$

$$
U_{t}-U^{*}\left(\sigma_{c}\right) \propto t^{-\delta} ; \delta=1
$$

Thus the democratic fiber bundles (for different fiber strength distributions) show phase transition with a well defined order parameter which shows similar power law variation on the way the critical point is approached. The susceptibility and relaxation time also diverge with same power exponent for all the cases. Therefore, failure of democratic fiber bundles belong to a universality class characterized by the universal values of the associated exponents $(\alpha, \beta, \gamma$ and $\delta)$.

\subsection{Nonlinear stress-strain relation}

One can now consider a slightly modified strength distribution of the democratic fiber bundle, showing nonlinear deformation characteristics ${ }^{3,6,11}$. For this, we consider an uniform density distribution of fiber strength, having a lower cutoff. Until failure of any of the fibers (due to this lower cutoff), the bundle shows linear elastic behavior. As soon as the fibers start failing, the stress-strain relationship becomes nonlinear. The dynamic critical behavior remains essentially the same and the static (fixed point) behavior shows elastic-plastic like deformation before rupture of the bundle.

Here the fibers are elastic in nature having identical force constant $\kappa$ and the random fiber strengths distributed uniformly in the interval $\left[\sigma_{L}, 1\right]$ with $\sigma_{L}>0$; the normalised distribution of the threshold stress of the fibers thus has the form (see Fig. 5):

$$
\rho\left(\sigma_{t h}\right)=\left\{\begin{array}{c}
0, \quad 0 \leq \sigma_{t h} \leq \sigma_{L} \\
\frac{1}{1-\sigma_{L}}, \sigma_{L}<\sigma_{t h} \leq 1
\end{array}\right.
$$




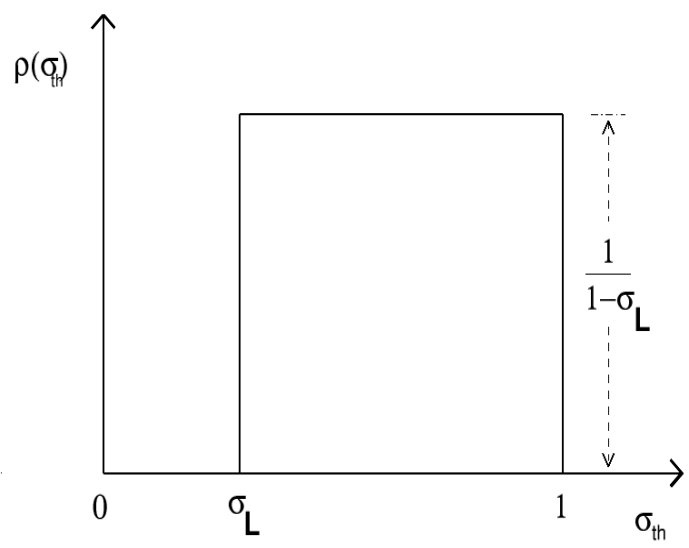

Fig. 5: The fiber breaking strength distribution $\rho\left(\sigma_{t h}\right)$ considered for studying elastic-plastic type nonlinear deformation behavior of the ELS model.

For an applied stress $\sigma \leq \sigma_{L}$ none of the fibers break, though they are elongated by an amount $\delta=\sigma / \kappa$. The dynamics of breaking starts when applied stress $\sigma$ becomes greater than $\sigma_{L}$. Now, for $\sigma>\sigma_{L}$ the fraction of unbroken fibers follows a recursion relation (for $\rho\left(\sigma_{t h}\right)$ as in Fig. 5):

$$
U_{t+1}=1-\left[\frac{F}{N U_{t}}-\sigma_{L}\right] \frac{1}{1-\sigma_{L}}=\frac{1}{1-\sigma_{L}}\left[1-\frac{\sigma}{U_{t}}\right],
$$

which has stable fixed points:

$$
U^{*}(\sigma)=\frac{1}{2\left(1-\sigma_{L}\right)}\left[1+\left(1-\frac{\sigma}{\sigma_{c}}\right)^{1 / 2}\right] ; \sigma_{c}=\frac{1}{4\left(1-\sigma_{L}\right)} .
$$

The model now has a critical point $\sigma_{c}=1 /\left[4\left(1-\sigma_{L}\right)\right]$ beyond which total failure of the bundle takes place. The above equation also requires that $\sigma_{L} \leq 1 / 2$ (to keep the fraction $\left.U^{*} \leq 1\right)$. As one can easily see, the dynamics of $U_{t}$ for $\sigma<\sigma_{c}$ and also at $\sigma=\sigma_{c}$ remains the same as discussed in the earlier section. At each fixed point there will be an equilibrium elongation $\delta(\sigma)$ and a corresponding stress $S=U^{*} \kappa \delta(\sigma)$ develops in the system (bundle). This $\delta(\sigma)$ can be easily expressed in terms of $U^{*}(\sigma)$. This requires the evaluation of $\sigma^{*}$, the internal stress per fiber developed at the fixed point, corresponding to the initial (external) stress $\sigma(=F / N)$ per fiber applied on the bundle when all the fibers were intact. From the first part of Eq. (36), one then gets $\left(\right.$ for $\sigma>\sigma_{L}$ )

$$
U^{*}(\sigma)=1-\frac{\sigma^{*}-\sigma_{L}}{\left(1-\sigma_{L}\right)}=\frac{1-\sigma^{*}}{1-\sigma_{L}}
$$

Consequently,

$$
\kappa \delta(\sigma)=\sigma^{*}=1-\left(1-\sigma_{L}\right) U^{*}(\sigma) .
$$


It may be noted that the internal stress $\sigma_{c}^{*}\left(=\sigma_{c} / U^{*}\left(\sigma_{c}\right)\right.$ from (2)) is universally equal to $1 / 2$ (independent of $\sigma_{L}$; from (36)) at the failure point $\sigma=\sigma_{c}$ of the bundle. This finally gives the stress-strain relation for the ELS model :

$$
S=\left\{\begin{array}{cc}
\kappa \delta, & 0 \leq \sigma \leq \sigma_{L} \\
\kappa \delta(1-\kappa \delta) /\left(1-\sigma_{L}\right), & \sigma_{L} \leq \sigma \leq \sigma_{c} \\
0, & \sigma>\sigma_{c}
\end{array} .\right.
$$

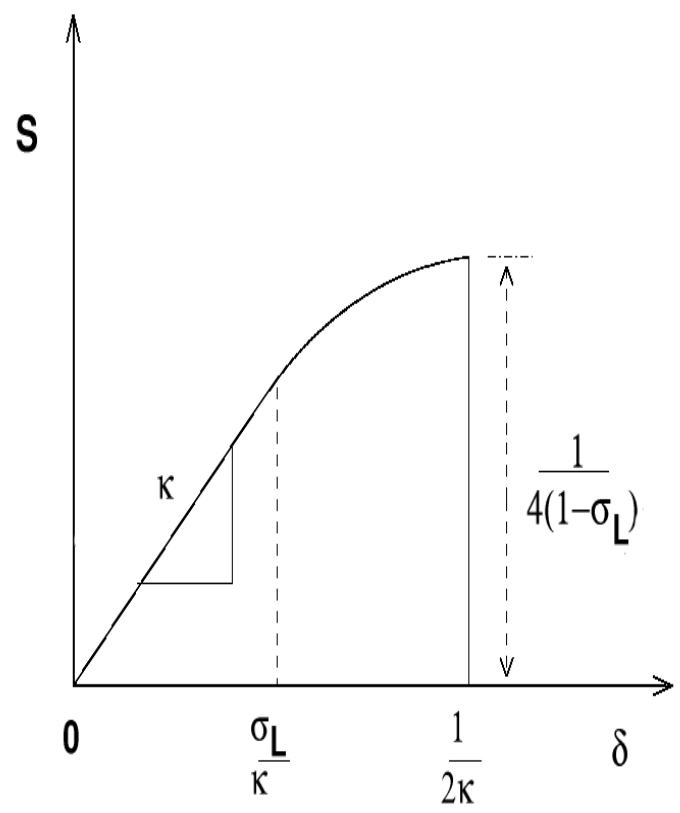

Fig. 6: Schematic stress $(S)$-strain $(\delta)$ curve of the bundle (shown by the solid line), following Eq. (39), with the fiber strength distribution (34) (as shown in Fig. 5).

This stress-strain relation is schematically shown in Fig. 6, where the initial linear region has slope $\kappa$ (the force constant of each fiber). This Hooke's region for stress $S$ continues up to the strain value $\delta=\sigma_{L} / \kappa$, until which no fibers break $\left(U^{*}(\sigma)=1\right)$. After this, nonlinearity appears due to the failure of a few of the fibers and the consequent decrease of $U^{*}(\sigma)$ (from unity). It finally drops to zero discontinuously by an amount $\sigma_{c}^{*} U^{*}\left(\sigma_{c}\right)=1 /\left[4\left(1-\sigma_{L}\right)\right]=\sigma_{c}$ at the breaking point $\sigma=\sigma_{c}$ or $\delta=\sigma_{c}^{*} / \kappa=1 / 2 \kappa$ for the bundle. This indicates that the stress drop at the final failure point of the bundle is related to the extent $\left(\sigma_{L}\right)$ of the linear region of the stress-strain curve of the same bundle. 


\subsection{Fatigue-failure in a homogeneous fiber bundle}

Fatigue in fiber bundle model was first studied by Coleman in $1958^{14}$. Thermally activated failures of fiber have recently been considered and approximate fatigue behavior has been studied ${ }^{16}$. We consider here a very simple fiber bundle model with noise-induced activated failure, for which the dynamics can be analytically solved.

Let us consider a homogeneous bundle of $N$ fibers under load $F(=N \sigma)$, each having identical failure strength $\sigma_{t h}$ which is the strength $\left(\sigma_{c}\right)$ of the bundle also. Without any noise $(\widetilde{T}=0)$, the model is trivial: the bundle does not fail (failure time $\tau$ is infinity) for stress $\sigma<\sigma_{c}$ and it fails immediately $(\tau=0)$ for $\sigma \geq \sigma_{c}$. We now assume that each such fiber has a finite probability $p(\sigma, \widetilde{T})$ of failure at any stress $\sigma$ induced by a non-zero noise $\widetilde{T}$ :

$$
p(\sigma, \widetilde{T})=\left\{\begin{array}{cc}
\frac{\sigma}{\sigma_{c}} \exp \left[-\frac{1}{\widetilde{T}}\left(\frac{\sigma_{c}}{\sigma}-1\right)\right], & 0 \leq \sigma \leq \sigma_{c} \\
1, & \sigma>\sigma_{c}
\end{array}\right.
$$

As one can see, each fiber now has got a non-vanishing probability $p(\sigma, \widetilde{T})$ to fail under a stress $\sigma<\sigma_{c}$ at any non-zero noise parameter $\widetilde{T} \cdot p(\sigma, \widetilde{T})$ increases as $\widetilde{T}$ increases and for $\sigma \geq \sigma_{c}, p(\sigma, \widetilde{T})=1$. Unlike at $\widetilde{T}=0$, the bundle therefore fails at $\sigma<\sigma_{c}$ after a finite time $\tau$. Here we assume each fiber to have unique threshold, while their breaking probability at any $\sigma\left(<\sigma_{c}\right)$ is due to noise-activated hopping over the barrier height $\left(\sigma_{c}-\sigma\right)$. This differs from the earlier model studies ${ }^{14,16}$ where the load distribution is noise induced.

\section{Failure time}

At $\widetilde{T} \neq 0$ and under any stress $\sigma\left(<\sigma_{c}\right)$, some fibers fail due to noise and the load gets shared among the surviving fibers, which in turn enhances their stress value, inducing further failure. Denoting the fraction of fibers to the initial bundle that remains intact at time $t$ by $U_{t}$, a discrete time recursion relation ${ }^{17}$ can be written as

$$
U_{t+1}=U_{t}\left[1-p\left(\frac{\sigma}{U_{t}}, \widetilde{T}\right)\right],
$$

where $\sigma / U_{t}=F /\left(N U_{t}\right)$ is the redistributed load per fiber among the $N U_{t}$ surviving fibers at time $t$. In the continuum limit, we can write the above recursion relation in a differential form

$$
-\frac{d U}{d t}=\frac{\sigma}{\sigma_{c}} \exp \left[-\frac{1}{\widetilde{T}}\left(\frac{\sigma_{c}}{\sigma} U-1\right)\right],
$$

The failure time $\tau$ is defined as $\tau=t$ when $U_{t}=0$. Integrating Eq. (42) within proper limits we get

$$
\tau=\int_{0}^{\tau} d t=\frac{\sigma_{c}}{\sigma} \exp \left(-\frac{1}{\widetilde{T}}\right) \int_{0}^{1} \exp \left[\frac{1}{\widetilde{T}}\left(\frac{\sigma_{c}}{\sigma}\right) U\right] d U
$$


or $^{17}$

$$
\tau=\widetilde{T} \exp \left(-\frac{1}{\widetilde{T}}\right)\left[\exp \left(\frac{\sigma_{c}}{\sigma \widetilde{T}}\right)-1\right]
$$

for $\sigma<\sigma_{c}$. For $\sigma \geq \sigma_{c}$, starting from $U_{t}=1$ at $t=0$, one gets $U_{t+1}=0$ from Eq. (41), giving $\tau=0$.

For small $\widetilde{T}$ and as $\sigma \rightarrow \sigma_{c}, \tau \simeq \widetilde{T} \exp \left[\left(\sigma_{c} / \sigma-1\right) / \widetilde{T}\right]$. This failure time $\tau$ therefore approaches infinity as $\widetilde{T} \rightarrow 0$. For $\sigma<\sigma_{c}$, one gets finite failure time $\tau$ which decreases exponentially as $\sigma$ approaches $\sigma_{c}$ or as $\widetilde{T}$ increases and $\tau=0$ for $\sigma \geq \sigma_{c}$. This last feature is absent in the earlier formulations ${ }^{16}$. However, all these features are very desirable and are in qualitative agreement with the recent experimental observations ${ }^{19}$. Our numerical study confirms the above analytic results [obtained using the continuum version of the recursion relation (41) (see Fig. 7) well.
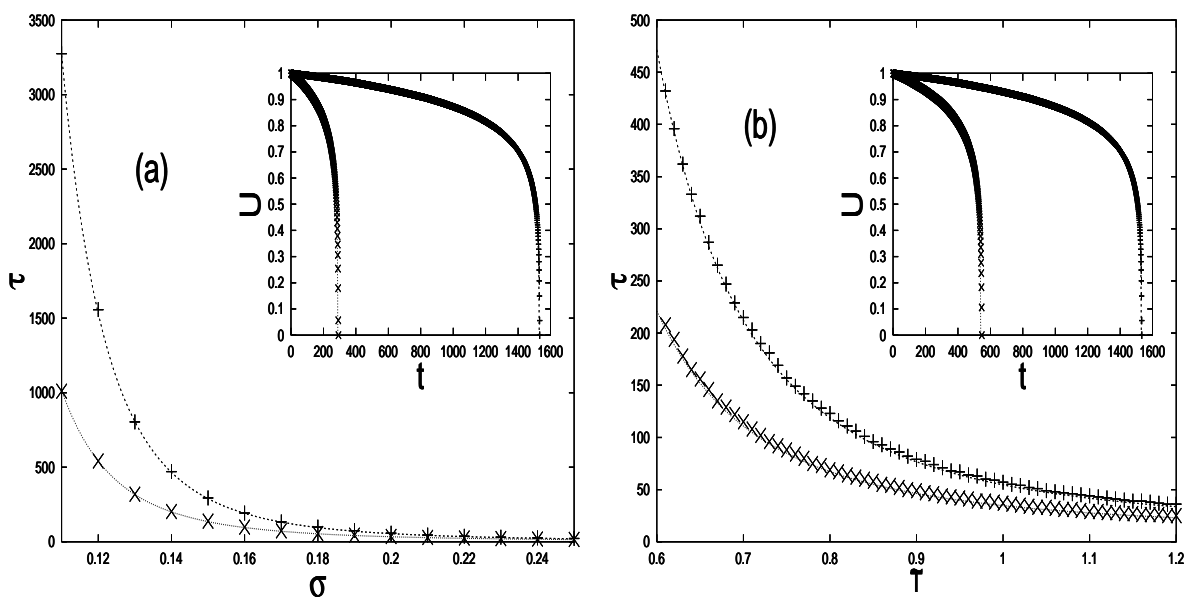

Fig. 7. The simulation results showing variation of average failure time $\tau$ against (a) stress $\sigma$ and (b) against noise $\widetilde{T}$, for a bundle containing $N=10^{5}$ fibers. The theoretical results are shown by dotted and dashed lines [from Eq. (44)]. The insets show the simulation results for the variation of the fraction $U$ of unbroken fibers with time $t$ for different $\widetilde{T}$ values [1.2 (cross) and 1.0 (plus)] in (a) and $\sigma$ values $[0.15$ (cross) and 0.12 (plus)] in (b). The dotted and dashed lines represent the theoretical results [Eq. (42)].

\section{Strength of the local load sharing (LLS) fiber bundles}

The strength of a bundle of fibers plays important role in the failure dynamics of the bundle when external load is applied. By a probabilistic approach Daniel ${ }^{3}$ pioneered the study of finding the strength distribution of a bundle in terms of strength of the constituent fibers. Daniel assumed equal sharing of applied load (ELS). Later, this work has been expanded in the context of material science ${ }^{4}$. This type of model shows (both analytically and numerically) existence of a critical 


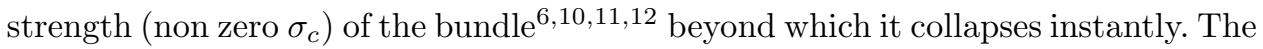
other extreme model, i,e., the local load sharing (LLS) model has been proved to be difficult to tackle analytically ${ }^{20}$. However some approximate asymptotic methods ${ }^{21}$ have been developed to tackle the problem in one dimension. Recently Pacheco et. al. ${ }^{22}$ introduced and solved the one sided load transfer model analytically. It is basically a simplification of the conventional (both sided) LLS model. Considering Weibull distribution of fiber strength, they obtained the system size dependence of the strength $\left(\sigma_{c}\right)$ of the bundle as

$$
\lim _{N \rightarrow \infty} \frac{1}{\sigma_{c}}=\text { const }+a_{s} \log _{2} N
$$

The subscript $s$ indicates the shape factor or Weibull index of the fiber strength distribution. This clearly shows $\sigma_{c} \rightarrow 0$ as $N \rightarrow \infty$. Smith ${ }^{23}$ conjectured a similar logarithmic dependence for LLS bundles from numerical results.

Here, we have simulated the above two types of LLS models: the one sided load transfer model and the conventional both sided load transfer model considering uniform distribution (random) of fiber strengths [Fig. 8]. We can not use the strictly uniform distrinution (as in Fig. 2), to avoid the increasing sequence of fiber strength arrangement which is fatal in case of LLS bundles though it does not matter in case of ELS bundles.

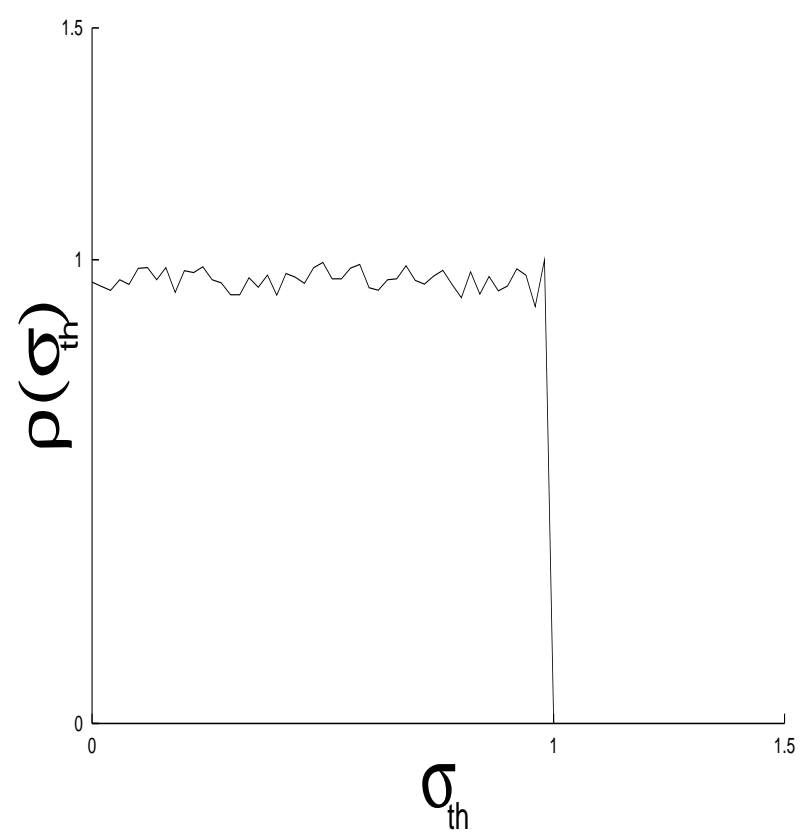

Fig. 8: The uniform (random) fiber strength distribution $\rho\left(\sigma_{t h}\right)$ considered to study the LLS models. 
We observe the $1 / \log N$ dependence of the bundle's strength $\left(\sigma_{c}\right)$ for both the cases [Fig. 9] which confirms the non existence of any critical strength (non zero $\sigma_{c}$ ) of the bundle in one dimension.

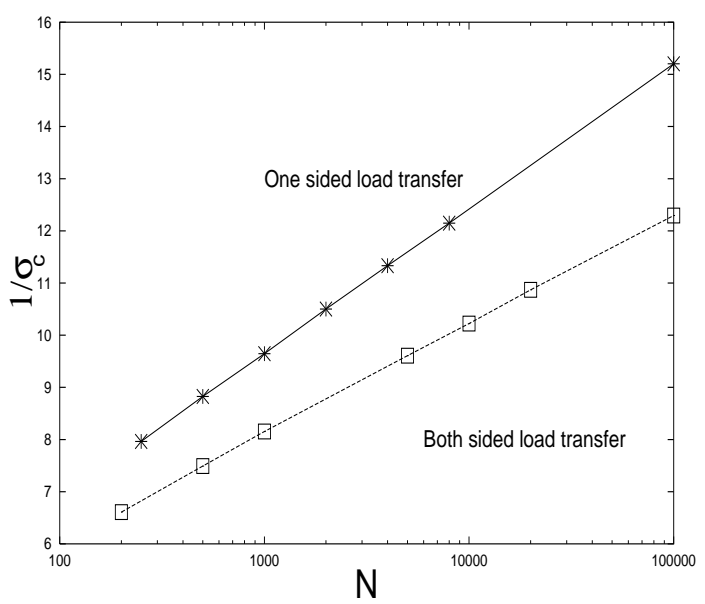

Fig. 9: The system size dependence of strength of the bundle $\left(\sigma_{c}\right)$ in LLS models. The results are obtained after averaging over $5 \times 10^{3}$ configurations.

\section{Magnetic mapping of the fiber bundle models}

We can map the fiber bundle problem in terms of a magnetic model or specifically an Ising model. The state of the fibers represent different states of the spins. We introduce a variable $u_{i}(t)$ to indicate the status of the $i$-th fiber at the time step $t$ such that $u_{i}(t)=1$ for intact fibers and 0 for broken fibers. The corresponding spin variable $s_{i}(t)=2 u_{i}(t)-1$ can then take two values \pm 1 , which can represent the up and down states of the Ising spin at $i$-th site. The strength threshold $\left(\sigma_{t h}\right)_{i}$ of the $i$-th fiber represents a random field at the $i$-th site (fiber) favoring $s_{i}=+1$ state (or $\left.u_{i}=1\right)$ and the stress $\sigma_{i}(t)$ on the $i$-th fiber represents a competing field at each site favoring the state $s_{i}=-1$ (or $u_{i}=0$ ). The value of $s_{i}(t)$ or $u_{i}(t)$ depends on the strength of the resultant field $h_{i}(t)$ :

$$
\begin{gathered}
s_{i}(t)=\operatorname{sgn}\left[h_{i}(t)\right] \\
h_{i}(t)=\left(\sigma_{t h}\right)_{i}-\sigma_{i}(t) ; u_{i}(t)=\frac{1}{2}\left[s_{i}(t)+1\right] .
\end{gathered}
$$

The only restriction on the competing fields is

$$
\sum_{i=1}^{N} u_{i}(t) \sigma_{i}(t)=\frac{1}{2}\left[s_{i}(t)+1\right] \sigma_{i}(t)=F ;
$$


$F$ is the applied force (load) on the bundle. $\sigma_{i}(t)$, and hence $h_{i}(t)$, evolves with time $t$ following the above relation. This is a general restriction for both, equal and local load sharing cases. The average magnetization at time $t$ can be defined as

$$
m(t)=\frac{1}{N} \sum_{i=1}^{N} s_{i}(t) .
$$

This non-interacting spin model is somewhat special as the zero-temperature dynamics of the spins here is determined by the strength of the effective feild $h_{i}$ at that site (46), where one part $\left(\sigma_{i}(t)\right)$ of $h_{i}$ evolves with time following (48). The stable manetization $m(=1$ at $\sigma=0)$ decreases continuously with $\sigma$ until it reaches a value $m_{c}=2 U^{*}-1$, where

$$
U^{*}=\frac{1}{N} \sum_{i=1}^{N} u_{i}(t \rightarrow \infty),
$$

at $\sigma=\sigma_{c}$, beyond which $m$ drops to -1 discontinuously.

For equal load sharing case, the above equation becomes

$$
\sigma(t) \sum_{i=1}^{N} u_{i}(t)=F=\sigma N ;
$$

$\sigma$ being the initial applied stress $(F / N)$. For uniform fiber strength distribution we have $U^{*}\left(\sigma_{c}\right)=1 / 2$ (from (10)). Here, $m_{c}=0$ and $m$ decreases continuously from unity (at $\sigma=0$ ) to $m=m_{c}=0$ at $\sigma=\sigma_{c}$ and then abruptly crosses over to $m=-1$ at $\sigma>\sigma_{c}$.

\section{Discussions}

The inherent mean-field nature of the ELS models enables to construct recursion relations (Eqs. 5 and 6) which captures essentially all the intriguing features of the failure dynamics. Though we have identified $O \equiv U^{*}(\sigma)-U^{*}\left(\sigma_{c}\right) \propto\left(\sigma_{c}-\sigma\right)^{\beta}$ as the order parameter (with exponent $\beta=1 / 2$ ) for the continuous transition in the ELS models, unlike in the conventional phase transitions it does not have a realvalued existence for $\sigma>\sigma_{c}$. The 'type' of phase transition in ELS models is still a controversial issue. Earlier ${ }^{7}$ it was suggested to be a first order phase transition, because the the surviving fraction of fibers has a discontinuity at the breakdown point of the bundles. However, as the susceptibility shows divergence $\left(\chi \propto\left(\sigma_{c}-\right.\right.$ $\left.\sigma)^{-\gamma} ; \gamma=1 / 2\right)$ at the breakdown point, the transition has been later identified to be of second order ${ }^{8,9,11,12}$. The dynamic critical behavior of the ELS models and the universality of the exponent values are straightforward. Here, divergence of relaxation time $(\tau)$ at the critical point $\left(\tau \propto\left(\sigma_{c}-\sigma\right)^{-\alpha} ; \alpha=1 / 2\right)$ indicates 'critical slowing' of the dynamics which is characteristic of conventional critical phenomena. At the critical point, one observes power law decay of the surviving fraction in time 
$\left(U_{t}\left(\sigma_{c}\right) \propto t^{-\delta} ; \delta=1\right)$. We demonstrated the universality of the failure behavior near $\sigma=\sigma_{c}$, for three different distributions: uniform (Fig. 2), linearly increasing (Fig. 3) and linearly decreasing (Fig. 4) distributions of fiber strength. The critical strengths of the bundles differ in each case: $\sigma_{c}=1 / 4, \sqrt{4 / 27}$ and $4 / 27$ respectively for these three distributions. However, the critical behavior of the order parameter $O$, susceptibility $\chi$, relaxation time $\tau$ and of the time decay at $\sigma_{c}$, as given by the exponents $\beta, \gamma, \alpha$ and $\delta$ remain unchanged: $\alpha=1 / 2=\beta=\gamma$ and $\delta=1$ for all three distributions.

The ELS model also shows realistic nonlinear deformation behavior with a shifted (by $\sigma_{L}$, away from the origin) uniform distribution of fiber strengths. The stress-strain curve for the model clearly shows three different regions: elastic or linear part (Hooke's region) when none of the fibers break $\left(U^{*}(\sigma)=1\right)$, plastic or nonlinear part due to the successive failure of the fibers $\left(U^{*}(\sigma)<1\right)$ and then finally the stress drops suddenly (due to the discontinuous drop in the fraction of surviving fibers from $U^{*}\left(\sigma_{c}\right)$ to zero) at the failure point $\sigma_{c}=1 /\left[4\left(1-\sigma_{L}\right)\right]$.

The fatigue study in a homogeneous fiber bundle suggests if the each fiber has a finite probability of failure (due to noise $\widetilde{T}$ as in (40)) below its normal strength, then the failure time of the bundle decreases exponentially $\left(\tau \simeq \widetilde{T} \exp \left[\left(\sigma_{c} / \sigma-1\right) / \widetilde{T}\right]\right)$ as $\sigma$ approaches $\sigma_{c}$ from below and $\tau \simeq 0$ for $\sigma>\sigma_{c}$. These features agree well with the experimental observations in disordered solids.

The LLS bundles show 'zero' critical strength as the bundle size goes to infinity in one dimension. It is not clear at this stage if, in higher dimensions, LLS bundles are going to have non-zero critical strength ${ }^{1,24}$. In any case, the associated dynamics of failure of these higher dimensional bundles with variable range load transfer ${ }^{13,25}$ should be interesting.

We believe, the elegance and simplicity of the model, its common-sense appeal, the exact solubility of its critical behavior in the mean field (ELS) limit, its demonstrated universality, etc, would promote the model eventually to a level competing with the Ising model of magnetic critical behavior.

\section{Acknowledgements}

We are grateful to Pratip Bhattacharyya for useful discussions. One of the author (BKC) is grateful to the selected High-School leaving students (in the Undergraduate Associateship Programme of the Saha Institute of Nuclear Physics) for their enthusiastic participation, comments and suggestions. Their involvement and appreciation encouraged the comparative view of the usefulness of the model over the Ising model discussed above. 


\section{References}

1. B. K. Chakrabarti and L. G. Benguigui, Statistical Physics of Fracture and Breakdown in Disorder Systems, Oxford Univ. Press, Oxford (1997), see also H. J. Herrmann and S. Roux (Eds), Statistical Models of Disordered Media, North Holland, Amsterdam (1990); P. Bak, How Nature Works, Oxford Univ. Press, Oxford (1997); M. Sahimi, Phys. Rep. 306, 213 (1980).

2. F. T. Peires, J. Textile Inst. 17, T355-368 (1926).

3. H. E. Daniels, Proc. R. Soc. London A 183405 (1945).

4. D. E. Gucer and J. Gurland, J. Mech. Phys. Solids 10, 365 (1962); S. L. Phoenix, Adv. Appl. Prob. 11, 153 (1979); R. L. Smith and S. L. Phoenix, J. Appl. Mech. 48, 75 (1981); R. L. Smith, Ann. Prob. 10, 137 (1982); L. N. McCartney and R. L. Smith, J. Appl. Mech. 50, 601 (1983).

5. P. C. Hemmer and A. Hansen, J. Appl. Mech. 59909 (1992); A. Hansen and P. C. Hemmer, Phys. Lett. A 184394 (1994); M. Kloster, A. Hansen and P. C. Hemmer, Phys. Rev. E 562615 (1997).

6. D. Sornette, J. Phys. A 22 L243 (1989); D. Sornette, J. Phys. I (France) 22089 (1992); A. T. Bernardes and J. G. Moreira, Phys. Rev. B 4915035 (1994).

7. S. Zapperi, P. Ray, H. E. Stanley and A. Vespignani, Phys. Rev. Lett. 781408 (1997);

8. Y. Moreno, J. B. Gomez and A. F. Pacheco, Phys. Rev. Lett. 852865 (2000).

9. R. da Silveria, Am. J. Phys. 671177 (1999).

10. S. Pradhan and B. K. Chakrabarti, Phys. Rev. E 65, 016113 (2001);

11. S. Pradhan, P. Bhattacharyya and B. K. Chakrabarti, Phys. Rev. E 66, 016116 (2002);

12. P. Bhattacharyya, S. Pradhan and B. K. Chakrabarti, Phys. Rev. E 67, 046122 (2003);

13. R. C. Hidalgo, F. Kun and H. J. Herrmann, Phys. Rev. E 64066122 (2001).

14. B. D. Coleman, J. Appl. Phys. 27, 862 (1956); B. D. Coleman, Trans. Soc. Rheol. 1, 153 (1957); B. D. Coleman, Trans. Soc. Rheol. 2, 195 (1958).

15. S. L. Phoenix, SIAM (Soc. Ind. Appl. Math.) J. Appl. Math. 34, 227 (1978); S. L. Phoenix, Adv. Appl. Probab. 11, 153 (1979); W. I. Newman and S. L. Phoenix, Phys. Rev. E 63, 021507 (2000); .

16. S. Roux, Phys. Rev. E 62, 6164 (2000); R. Scorretti, S. Ciliberto and A. Guarino, Europhys. Lett. 55, 626 (2001).

17. S. Pradhan and B. K. Chakrabarti, Phys. Rev. E 67, 046124 (2003).

18. L. Golubovic and S. Feng, Phys. Rev. A 43, 5223 (1991).

19. R. Banerjee and B. K. Chakrabarti, Bull. Mater. Sci. 24, 161 (2001); A. Guarino, S. Ciliberto, A. Garcimartin, M. Zei and R. Scorretti, cond-mat/0201257 (2002).

20. D. G. Harlow and S. L. Phoenix, J. Composit Mater. 12, 314 (1978); D. G. Harlow and S. L. Phoenix, Adv. Appl. Prob. 14, 68 (1982); R. L. Smith, Proc. R. Soc. London A 382, 179 (1982); R. L. Smith, Adv, Appl. Prob. 15, 304 (1982).

21. S. L. Phoenix and R. L. Smith, J. Solid Struct. 19, 479 (1983).

22. J. B. Gomez, D. Iniguez and A. F. Pacheco, Phys. Rev. Lett. 71, 380 (1993).

23. R. L. Smith, Proc. R. Soc. London A 372, 539 (1980).

24. G. Caldarelli and A. Petri, Phys. Rev. Lett. 83, 1483 (1999).

25. O. E. Yewande, Y. Moreno, F. Kun, R. C. Hidalgo and H. J. Herrman, cond mat/0210119 (2002). 